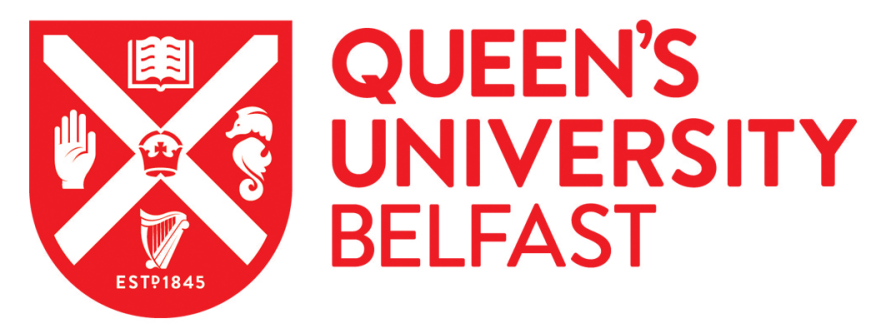

\title{
Strain dependent microstructural modifications of BiCrO3 epitaxial thin films
}

Kannan, V., Arredondo-Arechavala, M., Johann, F., Hesse, D., Labrugere, C., Maglione, M., \& Vrejoiu, I. (2013). Strain dependent microstructural modifications of BiCrO3 epitaxial thin films. Thin Solid Films, 545, $130-139$. https://doi.org/10.1016/j.tsf.2013.07.053

\section{Published in:}

Thin Solid Films

Document Version:

Peer reviewed version

Queen's University Belfast - Research Portal:

Link to publication record in Queen's University Belfast Research Portal

Publisher rights

(c) 2015, Elsevier. Licensed under the Creative Commons Attribution -NonCommercial-NoDerivs License

(https://creativecommons.org/licenses/by-nc-nd/4.0/), which permits distribution and reproduction for non-commercial purposes, provided the author and source are cited.

\section{General rights}

Copyright for the publications made accessible via the Queen's University Belfast Research Portal is retained by the author(s) and / or other copyright owners and it is a condition of accessing these publications that users recognise and abide by the legal requirements associated with these rights.

Take down policy

The Research Portal is Queen's institutional repository that provides access to Queen's research output. Every effort has been made to ensure that content in the Research Portal does not infringe any person's rights, or applicable UK laws. If you discover content in the Research Portal that you believe breaches copyright or violates any law, please contact openaccess@qub.ac.uk. 


\title{
Strain dependent microstructural modifications of $\mathrm{BiCrO}_{3}$ epitaxial thin films
}

\author{
Vijayanandhini Kannan ${ }^{1,2}$, Miryam Arredondo ${ }^{1}$, Florian Johann ${ }^{1}$, Dietrich Hesse ${ }^{1}$, \\ Christine Labrugere ${ }^{2,3}$, Mario Maglione ${ }^{2}$ and Ionela Vrejoiu ${ }^{1}$ \\ ${ }^{1}$ Max Planck Institute of Microstructure Physics, Weinberg 2, D-06120 Halle (Saale), \\ Germany. \\ ${ }^{2}$ CNRS, University of Bordeaux, ICMCB, UPR 9048, F-33600 Pessac, France. \\ ${ }^{3}$ CeCaMA, University of Bordeaux, ICMCB, F-33600 Pessac, France.
}

\begin{abstract}
Strain-dependent microstructural modifications were observed in epitaxial $\mathrm{BiCrO}_{3}$ (BCO) thin films fabricated on single crystalline substrates, utilizing pulsed laser deposition. The following conditions were employed to modify the epitaxial-strain: (i) in-plane tensile strain, BCOsтo [BCO grown on buffered $\left.\mathrm{SrTiO}_{3}(001)\right]$ and in-plane compressive strain, $\mathrm{BCO} \mathrm{NGO}\left[\mathrm{BCO}\right.$ grown on buffered $\left.\mathrm{NdGaO}_{3}(110)\right]$ and (ii) varying BCO film thickness. A combination of techniques like X-ray diffraction, X-ray photoelectron spectroscopy (XPS) and high resolution transmission electron microscopy (TEM) were used to analyse the epitaxial growth quality and the microstructure of BCO. Our studies revealed that in case of BCOsTo, a coherent interface with homogeneous orthorhombic phase is obtained only for BCO film with thicknesses, $d<50 \mathrm{~nm}$. All the BCOsto films with $d \gtrsim 50 \mathrm{~nm}$ were found to be strainrelaxed with an orthorhombic phase showing $1 / 2<100>$ and $1 / 4<101>$ satellite reflections, the latter oriented at $45^{\circ}$ from orthorhombic diffraction spots. High angle annular dark field scanning TEM of these films strongly suggested that the satellite reflections, $1 / 2<100>$ and $1 / 4<101>$, originate from the atomic stacking sequence changes (or "modulated structure") as reported for polytypes, without altering the chemical composition. The unaltered stoichiometry was confirmed by estimating both valency of $\mathrm{Bi}$ and $\mathrm{Cr}$ cations by surface and in-depth XPS analysis as well as the stoichiometric ratio (1 Bi: 1Cr) using scanning TEM - energy dispersive X-ray analysis. In contrast, compressively strained $\mathrm{BCO}_{\mathrm{NGO}}$ films exhibited monoclinic symmetry without any structural modulations or interfacial defects, up to $d \sim 200 \mathrm{~nm}$. Our results indicate that both the substrate-induced in-plane epitaxial strain and the BCO film thickness are the crucial parameters to stabilize a homogeneous BCO phase in an epitaxially grown film.
\end{abstract}




\section{Introduction}

Multiferroic oxides possessing more than one ferroic order parameter have promising properties, both for fundamental and technological aspects[

1]. These materials may be applied in integrated microelectronic devices, spintronic based magnetic field sensors, piezoelectric transducers, etc [2,3]. Among the known multiferroic materials, $\mathrm{Bi}$-based perovskite oxides, such as $\mathrm{BiMeO}_{3}(\mathrm{Me}=\mathrm{Fe}, \mathrm{Cr}, \mathrm{Mn}$, etc), have been extensively studied due to their high magnetic and ferroelectric (FE) ordering temperatures $[4,5]$. One of the above mentioned materials that possesses intrinsic multiferroic properties is $\mathrm{BiCrO}_{3}(\mathrm{BCO})[6,7,8]$. $\mathrm{BCO}$ has not been explored much yet, unlike $\mathrm{BiFeO}_{3}(\mathrm{BFO})$, which is quite appealing due to its large $\mathrm{FE}$ polarisation at room temperature (RT) $[9,10,11,12]$. In contrast to BFO, the existing literature on the synthesis and FE studies of BCO is very limited. Reports on the presence or absence of $\mathrm{FE}$ ordering in $\mathrm{BCO}$ at $\mathrm{RT}$ have led to controversial and ambiguous results $[13,14]$. Furthermore, enhanced $\mathrm{FE}$ and magnetic moments for the theoretically predicted $\mathrm{B}$-site ordered double perovskite, $\mathrm{Bi}_{2}(\mathrm{FeCr}) \mathrm{O}_{6}[15]$ have led to a great deal of experimental efforts to synthesize bulk and thin films of these compounds. In general, the synthesis of B-site ordered perovskites has been tackled by fabricating artificial superlattices or heterostructures, consisting of $\mathrm{BFO}$ and $\mathrm{BCO}$ thin films [16]. This has proven challenging, both in bulk and thin film form, mainly due to the difficulty in stabilising a phase pure $\mathrm{BCO}$ without predominating growth defects and coexistence of different crystallographic structures as reported in the literature. Phase pure BCO ceramics can be synthesized only by using high-pressure techniques, requiring about $6 \mathrm{GPa}[6]$. Some of the few reports on $\mathrm{BCO}$ are summarized here: (i) Belik et al. [17] have reported that bulk BCO ceramics exhibit a monoclinic $(\mathrm{C} 2 / \mathrm{c})$ crystal symmetry at RT, which transforms into an orthorhombic symmetry (Pnma) at 
around $420 \mathrm{~K}$. However, later, the same authors [18] noted that the monoclinic BCO phase retains about 10 to 15 volume percentage of an orthorhombic phase, suggesting multiple phase coexistence in BCO, (ii) Goujon et. al. [19] have pointed out the presence of $\sim 10 \mathrm{~nm}$ sized twinned domains within the monoclinic BCO phase, indicating its defective growth behaviour, (iii) Niitaka et. al. [20], in contrast, have suggested a distorted non-centrosymmetric crystal structure with C2 symmetry for BCO that could plausibly induce a FE structural distortion and (iv) David et al. [21] have recently shown that in case of BCO epitaxial thin films, an inhomogeneous strain distribution and oxygen-related defects within the BCO film can stabilize the coexistence of several distinct phases such as: monoclinic $(\mathrm{C} 2 / \mathrm{c})$ and orthorhombic (Pbnm), similar to the crystal structures already reported for bulk $\mathrm{BCO}$, and an "unknown" third phase giving rise to superlattice diffraction spots and whose crystal structure has not been identified so far. However, the nature and the origin of such multiple structural coexistences in BCO remain ambiguous.

Therefore, detailed knowledge in the crystal structure of BCO is fundamental to understand its FE properties [13,14], as well as studying B-site ordered multiferroic heterostructures based on $\mathrm{BCO}$ and $\mathrm{BFO}$ [15]. The present work focuses on the fabrication and microstructural analysis of BCO epitaxial thin films fabricated under different in-plane strain conditions, i.e., tensile and compressive strain. All the fabricated BCO films were investigated using a variety of techniques: X-ray diffraction (XRD), X-ray photoelectron spectroscopy (XPS), atomic force microscope (AFM) and high resolution transmission electron microscope (HRTEM). Our results show that the BCO films grown under an in-plane tensile and compressive strained conditions exhibit different crystallographic structures at RT. In addition, we found that in-plane tensile 
strained BCO thin films exhibit local structural modulations, directly correlated to the BCO layer thickness.

\section{Experimental details}

Epitaxial BCO thin films were fabricated by pulsed laser deposition (PLD) with a $\mathrm{KrF}$ excimer laser $(\lambda \sim 248 \mathrm{~nm})$ using a laser fluence of $\approx 2 \mathrm{~J} / \mathrm{cm}^{2}$. The BCO films were grown under an optimised conditions as follows: (i) substrate temperature $\left(T_{s}\right) \sim$ $650^{\circ} \mathrm{C}$ to $700^{\circ} \mathrm{C}$, (ii) oxygen partial pressure $\left(\mathrm{pO}_{2}\right) \sim 1.3$ to $2.6 \mathrm{~Pa}$ and (iii) laser frequency of 3 to $5 \mathrm{~Hz}$. Metallic SRO bottom electrodes (15 - $25 \mathrm{~nm}$ thick) were first deposited onto the substrates as a buffer layer, so that the BCO films could be subjected to electrical measurements in the future. Two different single crystalline and oriented substrates were used, to impose the following in-plane strain conditions, (i) tensile strain: BCO grown on $\mathrm{SrRuO}_{3}$ (SRO) buffered $\mathrm{SrTiO}_{3}$ (001) (STO) [BCOsTo] and (ii) compressive strain: $\mathrm{BCO}$ grown on $\mathrm{SRO}-$ buffered $\mathrm{NdGaO}_{3}(110)(\mathrm{NGO})$ [BCONGO]. The number line in Fig. 1(a) shows the pseudocubic in-plane lattice constants, $a_{\mathrm{pc}}$ (in $\AA$ ) for BCO, SRO, NGO (110) and STO (001) substrates [2,22]. The orthorhombic NGO (110) substrate (pseudo-cubic with $a_{\mathrm{pc}} \sim 3.867 \AA$ ) imposes an in-plane compressive strain of approx. $-1.08 \%$, whereas STO (001) (acubic $\sim 3.905 \AA$ ) imposes an in-plane tensile strain $(+0.64 \%)$ on BCO. To achieve atomically flat terraces having a single unit cell step height, both types of substrates were pre-treated: NGO (110) was thermally annealed at $1000^{\circ} \mathrm{C}$ for $2 \mathrm{~h}$, whereas STO (001) was chemically etched using a NH4F-buffered $\mathrm{HF}$ solution followed by thermal annealing $\left(950^{\circ} \mathrm{C}\right.$ to $1000^{\circ} \mathrm{C}$ for $\left.2 \mathrm{~h}\right)$. Both $\mathrm{BCO}_{\mathrm{sTO}}$ and $\mathrm{BCO}_{\mathrm{NGO}}$ films were fabricated under similar growth conditions (with various thickness of BCO film, $d$ ranging from 10 to $200 \mathrm{~nm}$ ). Some of the BCOsto films $(d>50 \mathrm{~nm})$ were subjected to post deposition annealing at higher oxygen partial 
pressures. Surface morphology of the fabricated films was investigated using an AFM (DI 5000, Veeco) operated in the tapping mode. The surface topography of the pretreated STO (001) is displayed in Fig. 1(b). The surface morphology of the SRO-bottom electrode fabricated onto STO (001), followed the step and terrace-like substrate morphology, indicating that step-flow growth took place [Fig. 1(c)]. The crystallinity and epitaxial nature of all the BCO films were analysed by high resolution XRD studies (Philips X' Pert MRD, $\mathrm{Cu} \mathrm{K}_{\alpha}$ radiation). A VG Scientific 220 i-XL ESCALAB spectrometer was used for surface and in-depth XPS analysis with a monochromatized $\mathrm{AlK}_{\alpha}$ source $(\mathrm{h} v=1486.6 \mathrm{eV})$ at $70 \mathrm{~W}$. The spot size was about $180 \mu \mathrm{m}$ in diameter and a pressure of $10^{-7} \mathrm{~Pa}$ was maintained in the chamber during analysis. The full spectra (0-1350 eV) were obtained with constant pass energy of $150 \mathrm{eV}$ and high resolution spectra with constant pass energy of $40 \mathrm{eV}$. High resolution XPS spectra were fitted and quantified using the AVANTAGE software provided by ThermoFisher Scientific. Microstructural data were investigated using TEM (JEOL 4010 at $400 \mathrm{kV}$ ) and high angle annular dark field scanning transmission electron microscopy (HAADF-STEM)

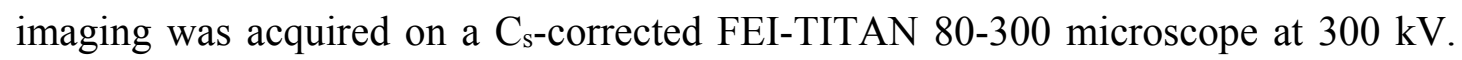
Both cross-sectional and plan-view TEM samples were prepared by standard mechanical and ion beam polishing procedures [23]. The stoichiometric ratios of cations (1Bi:1Cr) were confirmed for both BCOsto and BCONGO films, by STEM energy dispersive X-ray (EDX) line scans; excluding the possibilities of Bi volatility under the present growth conditions (not shown here).

\section{Results and discussion}

The representative XRD data for the $\mathrm{BCO}_{\mathrm{sto}}(d \sim 85 \mathrm{~nm})$ and $\mathrm{BCO}_{\mathrm{NGO}}(d \sim 160$ nm) samples is displayed in Figs. 2(a) and 2(b), respectively. Phase pure and highly oriented epitaxial growth could be envisaged for both the films, without any minor 
secondary phases of $\mathrm{Bi}_{2} \mathrm{O}_{3}$ or $\mathrm{Cr}_{2} \mathrm{O}_{3}$, etc. The reflections around $\mathrm{STO}(003)$ and $\mathrm{NGO}(330)$ are enlarged as inset in Figs. 2(a) and 2(b), respectively, to clearly reveal the presence of close peak splitting corresponding to the $\mathrm{BCO}$ and SRO phases, which indicates an oriented growth with their c-axis normal to STO. In addition, the BCONGO XRD exhibit a minor peak (*), possibly arising due to the misaligned grains of the perovskite structure with different orientation during strain relaxation [Fig. 2(b)]. The out-of-plane lattice constant for both $\mathrm{BCO}_{\mathrm{STO}}$ and $\mathrm{BCO}_{\mathrm{NGO}}$ from the $\theta / 2 \theta$ XRD scan is $\sim 3.88 \AA$ and $3.9 \AA$ respectively, in agreement with the reported values of BCO epilayers [18]. The rocking curve analysis performed along the (004) reflection (not shown here) displayed a full width at half maximum of approx. $0.2^{\circ}$, indicating a textured-type growth nature for all BCO films fabricated onto the STO and NGO substrates. The epitaxial growth of BCO films was further confirmed using the conventional $\Phi-$-scan analysis that clearly indicated a cube-on-cube growth of BCO and the SRO buffer layer onto both types of substrates.

Reciprocal space mapping (RSM) analysis was performed to reveal the nature of lattice misfit strain and to estimate the in- and out-of-plane lattice constants. Figures 3(a) - 3(d) depict the RSM images recorded along the asymmetric (203) reflection for both $\mathrm{BCO}_{\text {sto }}$ and $\mathrm{BCO}_{\mathrm{NGO}}$ films, respectively. The RSM plot for BCOsto does not exhibit a distinct, separate (203) peak for BCO [Fig. 3(a)]. Instead, the BCO (203) reflection is shown as a broad peak that is closely located around the STO (203) peak, thus revealing a good lattice constant matching with STO. The intensity and broadness of the BCO (203) reflection decreased with the thickness of the BCO layer [Figs. 3(a) - 3(c)]. A coherent and strained growth of BCO could be envisaged only for the layer thicknesses below $50 \mathrm{~nm}$, as depicted in Fig. 3(c). The nearly circular peak above the STO reflection in Figs. 3 (a) -3 (c) corresponds to SRO (203), which indicates a 
strained and coherent growth of the SRO buffer layer of less than $25 \mathrm{~nm}$ thickness. Figure 3(d) depicts the RSM analysis of a $\mathrm{BCO}_{\mathrm{NGO}}$ film. The RSM image clearly revealed a partial strain relaxation for the studied $\mathrm{BCO}_{\mathrm{NGO}}$ film with thickness, $d \sim 160$ $\mathrm{nm}$ and also revealed the splitting of the BCO (203) peak corresponding to the monoclinic-type distortion. This type of monoclinic splitting is very similar to that reported for compressively strained $\mathrm{BiFeO}_{3}$ epitaxial thin films [24]. The RSM analysis revealed that the out-of-plane and in-plane lattice constants for the BCONGO film adopt pseudocubic values of approx. $3.9 \AA$ and $3.87 \AA$, respectively, in correlation with the $\theta / 2 \theta$ XRD scan

Figure 4(a) is the AFM topography image for BCOsто, $(d \sim 85 \mathrm{~nm})$ which shows smooth and dense morphological features without any pore or crack formation at the surface; the RMS roughness value is $0.7 \mathrm{~nm}$. In addition, this AFM image reveals an interesting surface structure with micron-sized rectangular blocks having two different azimuthal orientations perpendicular to each other. Inside these rectangular blocks, stripe-like features are clearly observed that could possibly be due to the texture-like epitaxial growth. In fact, the size of these rectangular blocks decreased with decreasing film thickness for $\mathrm{BCO}_{\text {sto. }}$ Figure 4(b) depicts the AFM image for a $\mathrm{BCO}_{\mathrm{NGO}}$ film $(d$ $\sim 160 \mathrm{~nm}$ ), whose surface morphological features differed much from that of BCOsтo films, since it revealed a dense and grainy characteristics. This is probably due to the morphology of the bottom electrode (SRO), which did not grow in step-flow regime on NGO substrates. The RMS roughness for the $\mathrm{BCO}_{\mathrm{NGO}}$ film was found to be below 0.8 nm.

An XPS analysis was carried out for one of the BCOsto $(d \sim 100 \mathrm{~nm}$ thick $)$ films, to analyse the cationic valency and the oxygen homogeneity both from the $\mathrm{BCO}$ 
film's surface to the depth across the $\mathrm{BCO} / \mathrm{SRO}$ and $\mathrm{SRO} / \mathrm{STO}$ interfaces. The in-depth XPS profiling was performed by combining a sequence of $\mathrm{Ar}^{+}$ion etching followed by the XPS data acquisition from the etched surface. Figure 5(a) - 5(c) depicts the high resolution XPS spectra of $\mathrm{Bi} 4 f, \mathrm{Cr} 2 p$ and $\mathrm{O}$ 1s core levels respectively. The $\mathrm{Bi} 4 f$ photoemission spectra exhibited two peaks at the binding energy (B.E) of $159.1 \mathrm{eV}$ and $164.4 \mathrm{eV}$ corresponding to $4 f_{7 / 2}$ to $4 f_{5 / 2}$ transition of $\mathrm{Bi}^{3+}$ [25]. However, additional shoulder peaks (marked by arrows) associated with the major Bi $4 f$ peaks at the lower B.E values of 157.3 and $162.5 \mathrm{eV}$, emerge due to the partial Bi reduction via breakage of Bi-O bonds and oxygen dissociation during the Ar+ sputtering that leads to the metallic Bi bonding [Fig. 5(a) inset]. The Cr $2 p$ core level spectra displayed two peaks at B.E $\sim 576.7 \mathrm{eV}$ and $586.6 \mathrm{eV}$, which are attributed to the $2 p_{3 / 2}$ and $2 p_{1 / 2}$ excitation levels, respectively [Fig. 5(b)]. The estimated B.E peak positions for both $\mathrm{Bi} 4 f$ and $\mathrm{Cr}$ $2 p$ levels are in correlation with the reported values for $\mathrm{Bi}^{3+}$ and $\mathrm{Cr}^{3+}$ ions in several perovskites like $\mathrm{BiFeO}_{3}, \mathrm{Bi}_{4} \mathrm{Ti}_{3} \mathrm{O}_{12}, \mathrm{LaCrO}_{3},\left(\mathrm{La}_{1-\mathrm{x}} \mathrm{Sr}_{\mathrm{x}}\right) \mathrm{CrO}_{3}$ or $\left(\mathrm{Pr}_{1-\mathrm{x}} \mathrm{Ca}_{\mathrm{x}}\right) \mathrm{CrO}_{3}$, etc. [26, $27,28]$, thus suggesting a +3 oxidation states for all the $\mathrm{Bi}$ and $\mathrm{Cr}$ ions in the $\mathrm{BCO}$ film. Similarly, Fig. 5(c) depicts the in-depth O 1s core level spectra which exhibit the B.E peak at $\sim 530.3 \mathrm{eV}$. The peak positions and asymmetries of various core level spectra were analyzed after the Gaussian fittings as shown in Fig. 5(d)-5(f) [for sputter depth of $22 \mathrm{~nm}$ from BCO surface]. The analysis of the peak intensity and positions for all the fitted $\mathrm{Bi} 4 f, \mathrm{Cr} 2 p$ and $\mathrm{O} 1 s$ core level spectra at different $\mathrm{BCO}$ film depths suggested homogeneous oxygen distribution excluding the possibilities of multiple oxidation states for $\mathrm{Cr}$ or $\mathrm{Bi}$ ions as well as the secondary phase segregation. Further, the in-depth XPS analysis for this $\mathrm{BCO}_{\text {sto }}$ sample showed a nearly constant ratio of $\mathrm{Bi}, \mathrm{Cr}$ and oxygen contents (atomic \%) throughout the $100 \mathrm{~nm}$ depth of the heterostructure, beyond which the XPS signals of $\mathrm{Sr} 3 d$ and $\mathrm{Ru} 3 d$ core level signals corresponding to 
the SRO buffer layer dominated [Fig. 6]. In-depth XPS data was performed until the STO substrate surface was reached, wherein, high Sr and Ti core level signals were obtained.

Figure 7(a) is a bright field (BF) TEM image displaying an overview for a $d \sim$ $130 \mathrm{~nm}$ thick BCOsто cross-section sample. The inset in Fig. 7(a) corresponds to the selected area electron diffraction (SAED) pattern acquired for the whole BCOsTo heterostructure along the $[001]$ sto zone axis. Analysis of the main SAED pattern revealed that the BCOsто film exhibit an orthorhombic crystal structure and its estimated pseudo-cubic lattice constants revealed that the BCOsто film experiences an in-plane lattice tensile strain. Further, the epitaxial relationship is (100)STO $\|(100) \mathrm{SRO}\|(101) \mathrm{BCO}_{\text {ortho }}$ and [001]STO $\|[001] \mathrm{SRO}\|[010] \mathrm{BCO} \mathrm{ortho}_{\text {, }}$ indicating a coherent "cube on cube" growth of the pseudocubically considered BCO layer on SRO-buffered STO. The BF image showed significant changes in contrast within the $\mathrm{BCO}$ film, which suggests a strained or defective growth of the $\mathrm{BCO}$ phase (see area marked by the white box). To analyse these regions, HRTEM images were acquired from areas such as the one highlighted by the white box in Fig. 7(a) and shown in Fig. 7(b). Several Fast Fourier transforms (FFT) were calculated from within the HRTEM image. Figures 7(c) and 7(d) display the FFTs calculated from those areas highlighted as 1 and 2, respectively in Fig. 7(b). Figure 7(c) shows the main BCO orthorhombic [010] unit mesh with $1 / 2$ satellite reflections (indicated by arrows) along $<100>$. The presence of such satellite reflections indicates a possible cell doubling with a supercell parameter of $7.8 \AA$. Figure 7(d) indicates the [010] main orthorhombic unit mesh, similar to that of Fig. 7(c), but with $1 / 4$ satellite reflections appearing at a diagonal $\left(45^{\circ}\right)$ with respect to the orthorhombic diffraction spots along $<101>$ (as marked by arrows). The FFT patterns possessing satellite reflections strongly indicated the 
possibility of structural modulations within the main orthorhombic phase for the 130 nm thick BCOsтo film.

A plan-view TEM analysis was carried out for this BCOsto $(d \sim 130 \mathrm{~nm})$ film.

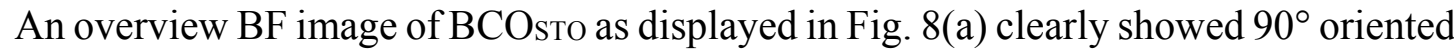
texture-like growth features whose averaged size is in correlation with the rectangular blocks observed in the corresponding AFM image. The SAED pattern in Fig. 8(b) exhibits a square perovskite structure which is associated with presence of extra satellite reflections (similar to those shown in Fig. 7) along two perpendicular crystallographic directions, wherein, the later originate due to the presence of twinned lattice planes. Further, the HAADF-STEM was used to characterise the apparently defective regions as observed by cross-sectional TEM and the BCO/SRO interface. From the overview of the BCOsтo film [Fig. 9(a)] the contrast variations, previously seen in Fig. 7, were not present and, more importantly, it should be noted that no change in the crystal structure of $\mathrm{BCO}$ was evident from our high-resolution HAADF imaging. The latter gives an indication that the defective areas observed in cross-sectional TEM (Fig. 7) originate from the modulated lattice structure. Further, the BCO layer of this sample exhibits defect lines perpendicular to the $\mathrm{BCO} / \mathrm{SRO}$ interface, as marked by the white boxes in Fig. 9(a). Figure 9(b) is a zoomed-in image of the area marked as "1" in Fig. 9(a) to demonstrate that the defective line is formed by four edge-type dislocations originating from the $\mathrm{BCO} / \mathrm{SRO}$ interface. Figure $9(\mathrm{c})$ is a high-resolution HAADF image of the top dislocation in Fig. 9(b), that shows its Burgers circuit with a vector of $\mathrm{b}=\mathrm{a}[101]$. The edge-type dislocation displayed in Fig. 9(c) is representative for all dislocations observed in different areas of the BCOsto film. 
To investigate the possibility of structural modulation within the orthorhombic BCO phase, a detailed analysis using HAADF-STEM imaging was performed on the cross-sectional and planar view samples (not shown) for $d \sim 130 \mathrm{~nm}$ thick BCOsтo film. Figure 10(a) depicts a high-resolution HAADF image extracted from the top of the BCOsтo layer, wherein no apparent structural differences could be observed. We point out that although the HAADF parameters were set to reach Z-contrast, contributions from the B-site $(\mathrm{Cr})$ ions were not evident, possibly due to the large difference in atomic numbers of $\mathrm{Cr}$ and $\mathrm{Bi}$ and also the large thickness of the BCOsto film. However, its corresponding FFT pattern (inset) revealed $45^{\circ}$ oriented satellite reflections, similar to those observed from the SAED patterns of the cross-sectional TEM. Furthermore, the HAADF image was processed using Digital Micrograph software to filter and create two lattice images by selecting (i) the main reflections (as marked by the red circle in the inset) and (ii) $45^{\circ}$ oriented satellite reflections (as marked by the blue circles). The resulting lattice images are displayed in Figs. 10(b) and 10(c), respectively, both with their corresponding zoomed-in views as an inset. In Fig. 10(b), a cubic lattice pattern is observed, similar to the original HAADF image which is expected to arise from the A site cation (Bi). However, Fig. 10(c) revealed a hexagonal-like pattern for all its structure. Next, in Fig. 10(d), these two lattice images are combined to obtain a false colored image, wherein, the red color is assigned to the main diffraction spots and blue for satellite reflections. It is evident that the two lattice images corresponding to the main orthorhombic lattice and the extra diffraction spots do not superimpose, but rather occupy different lattice positions, suggesting no crystal structural changes. From this analysis, we conclude that within the orthorhombic BCOsтo phase, there exists a modulated hexagonal-like lattice pattern that possibly arises from the $\mathrm{Cr}-\mathrm{O}$ sublattice. Further, such a lattice structural modulation gives rise to the $45^{\circ}$ oriented $1 / 2$ and $1 / 4$ extra 
superlattice reflections. The latter can be attributed to an "intergrowth" or "modulated structure", i.e. stacking sequence variations similar to those seen in polytypes. Polytype structures are considered to arise from different stacking of atomic layers within the same crystal structure and with no change in overall chemical composition [24]. Formations of such polytypes have been reported in several alkaline-earth perovskites like $\mathrm{BaCrO}_{3}$ [29], $\mathrm{BaRuO}_{3}$, etc. [30,31], but, so far, no such report exists in case of the Bi-based perovskite oxides. Further, there have been ample evidences from the earlier studies [28-30] that the polytypes are revealed by extra satellite reflections and/or streak formations in the SAED pattern [32]. Moreover, this will explain why no structural changes are observed in our HAADF images.

Changes in microstructure with respect to the layer thickness were observed for BCOsto film thicknesses varying from 10 to $130 \mathrm{~nm}$ (Fig. 11). Here we show the TEM data only for the 85 and $25 \mathrm{~nm}$ thick BCOsto films [Figs. 11(a) and 11(c)]. The BF image of the $85 \mathrm{~nm}$ thick BCOsтo film exhibited in the vicinity of the top surface several areas with dark contrast, probably strained regions (marked by a circle) in addition to the presence of edge-type dislocations that originate at the $\mathrm{BCO} / \mathrm{SRO}$ interface, similar to those of Fig. 9(a). All the BCOsto films grown with thickness below $d \approx 50 \mathrm{~nm}$ do not exhibit any evidences of edge-type dislocations. As displayed in Fig. 11(c), a coherent growth and sharp BCO/SRO interface without any contrast variation were observed for all these films. The analysis of the SAED patterns acquired from the whole sample in the case of the thicker BCO sто $_{\text {[Fig. 11(b)] strongly suggested }}$ that the strained dark contrast regions were responsible for the satellite diffraction spots (marked by arrows), indicating a structural modulation. This was seen only for BCOsто films of thicknesses $d \gtrsim 50 \mathrm{~nm}$ that are strain relaxed. For BCOsтo samples with BCO 
layers below $50 \mathrm{~nm}$ thickness, the strained dark contrast regions disappeared and the edge-type dislocation density considerably decreased. They exhibited a homogeneous orthorhombic crystal structure, observed in its SAED [Fig. 11(d)]. A similar TEM analysis was carried out for BCOSTo films that were subjected to post deposition annealing treatments at higher oxygen partial pressures. We observed no significant modification in the microstructure due to post deposition oxygen annealing (not shown here).

To unravel the role of epitaxial strain on the structural modulation, BCO films were grown on an orthorhombic $\mathrm{NdGaO}_{3}(110)$ [NGO] substrate with and without the SRO buffer layer. A large thickness ( $d$ up to $200 \mathrm{~nm}$ ) of $\mathrm{BCO}_{\mathrm{NGO}}$ thin films was particularly chosen to verify whether the compressive epitaxial strain relaxation stabilises a homogeneous BCO phase without any structural modulation. Figure 12 displays the TEM data set for a $160 \mathrm{~nm}$ thick BCO film fabricated onto $15 \mathrm{~nm}$ thick SRO buffered NGO (110) substrate. The high-resolution HAADF image [Fig. 12(a \& 12b)] shows an overview of the sample, wherein both the BCO/SRO and SRO/STO interfaces appear smooth. Thus, our TEM studies suggest that the thick and strainrelaxed BCONGO film does not exhibit any extended defects such as dislocations or twinning or the spacing between the dislocations must be quite large. Figure 12(c) is the SAED pattern for the $\mathrm{BCO}_{\mathrm{NGO}}$ sample, which indicates the epitaxial growth and more importantly no extra superlattice diffraction spots ("structural modulation") could be observed. Indexing of the SAED pattern showed that the compressively strained $\mathrm{BCO}_{\mathrm{NGO}}$ films exhibit a monoclinic crystal structure with an epitaxial relationship: (110)NGO $\|(100) \mathrm{SRO} / \mathrm{BCO}$ and $[011] \mathrm{NGO} \|[001] \mathrm{SRO} / \mathrm{BCO}$. The monoclinic BCONGO films, fabricated up to $d \sim 200 \mathrm{~nm}$, exhibited no structural modulations, in 
contrast to the BCOsто $(d>50 \mathrm{~nm})$ films. In addition, the presence of the SRO buffer layer up to thickness $\leq 25 \mathrm{~nm}$, seems to play an insignificant role in altering the epitaxial strain on the BCO films, in case of both STO and NGO substrates.

In summary, at the macroscale, our structural and chemical analysis of both $\mathrm{BCO}_{\text {sto }}$ and $\mathrm{BCO}_{\mathrm{NGO}}$ films, using the high resolution $\theta / 2 \theta$ XRD, RSM (203) scan, XPS and STEM-EDX analysis, respectively, revealed a highly oriented epitaxial growth with a stoichiometric cationic ratio $(1 \mathrm{Bi}: 1 \mathrm{Cr})$ and no oxygen inhomogeniety both at the film's surface as well as at the cross-sectional regions of the BCO heterostructure. In contrast, our TEM studies showed some interesting results. The crystallographic phase content of the tensile strained BCOsto films was found to be an orthorhombic structure, whereas the $\mathrm{BCO}_{\mathrm{NGO}}$ films exhibit a monoclinic symmetry. Thus, in contrast to the previous reports on multiple phase coexistence within the $\mathrm{BCO}$ bulk [17-20] or thin films [21], the present work suggests a variation in crystal structure, depending on whether the $\mathrm{BCO}$ films experienced tensile (BCOsto) or compressive $\left(\mathrm{BCO}_{\mathrm{NGO}}\right)$ inplane strain. The tensile-strained orthorhombic BCOsтo films of thicknesses, $d \gtrsim 50$ $\mathrm{nm}$ exhibit a high density of edge-type dislocations originating near the $\mathrm{BCO} / \mathrm{SRO}$ interface, which could be attributed to the semicoherent growth and an epitaxial strain relaxation. The HAADF-STEM analysis of those strain relaxed BCOsto $(d \gtrsim 50 \mathrm{~nm})$ films with $1 / 2<100>$ and $1 / 4<101>$ extra satellite reflections suggested changes in the atomic lattice sequences or modulation similar to polytypes [24]. We point out that only those strain relaxed BCOsтo films exhibit extra satellite diffraction spots or structural modulation within the main orthorhombic phase. Further, the epitaxial strain modification via the layer thickness suggested that the presence of structural modulation critically depends on the BCO film thickness and it is thus inevitable to 
limit its thickness, $d$ below $50 \mathrm{~nm}$, in order to stabilise a homogeneous orthorhombic phase. Such films would grow coherently with least interfacial defects.

The in-plane epitaxial strain was further altered by fabricating compressively strained BCO films on NGO (110) substrate with or without SRO buffer layer. Our TEM studies showed that $\mathrm{BCO}_{\mathrm{NGO}}$ films with $d$ up to $200 \mathrm{~nm}$ grown under an in-plane compressive strain have a monoclinic crystal structure. Although, the RSM analysis of

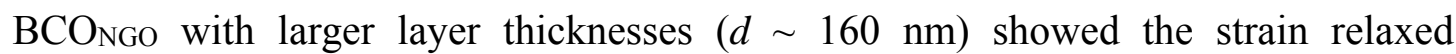
monoclinic (203) peak splitting [Fig. 3(d)], we do not observe any defective BCO/SRO interfaces in HRTEM. For BCONGO films, the interfacial regions analysed using STEMHAADF also showed a coherent interface without any structural defects as well as misfit or edge-type dislocations. More importantly, compressively strained BCONGO films exhibit no superstructure diffraction spots in SAED patterns unlike the tensile strained BCOsto films. All these results suggest that in case of compressive strain induced by NGO (110) substrate, the strain relaxation of BCO does not induce any structural modulation. We note in this context that, as suggested by David et al. [21] the inhomogeneous strain distribution or relaxation would lead to the prevalence of structural modulations in case of the tensile strained BCOsтo films.

\section{Conclusions}

Growing non-defective and relatively thick BCO epitaxial films that possess homogeneous crystal structure is a prerequisite to study their electrical properties, eliminating the large contributions from leakage current. The present study showed that it is possible to stabilise an epitaxial $\mathrm{BiCrO}_{3}$, one of the metastable phases of $\mathrm{Bi}$-based multiferroic oxides by PLD utilizing the substrate induced strain. Furthermore, an 
appropriate choice of the substrate to fabricate an in-plane strained epitaxial BCO film as well as its thickness (in case of BCOsто) are crucial parameters to obtain either an orthorhombic or monoclinic phase, and also may give insight into the fabrication of Bsite ordered BCO superlattices or heterostructures with other isostructural compounds like $\mathrm{BiFeO}_{3}, \mathrm{BiMnO}_{3}$ etc.

\section{Acknowledgement}

This work was supported by the CNRS-MPG joint fellowship programme on nanoscience. V. K. would like to thank Dr. R. Ranjith and Dr. Wei Peng for the scientific discussions. The authors also acknowledge Mr. Schammelt for PLD lab maintenance, Mareike Herrmann for TEM sample preparations and Dr. Eckhard Pippel for STEM-EDX analysis.

\section{References}

[1] M. Mostovoy, Nature Mater. 9 (2010) 188.

[2] L.W. Martin, Y. H. Chu, R. Ramesh, Mater. Sci. Eng. R 68, (2010) 89.

[3] D. Budker, Nature Mater. 9 (2010) 608. 
[4] J. Wang, J. B. Neaton, H. Zheng, V. Nagarajan, S. B. Ogale, B. Liu, D. Viehland, V. Vaithyanathan, D. G. Schlom, U. V. Waghmare, N. A. Spaldin, K. M. Rabe, M. Wuttig, R. Ramesh, Science 29 (2003)1719.

[5] K. Vijayanandhini, C. Simon, V. Pralong, Y. Bréard, V. Caignaert, B. Raveau, P. Mandal, A. Sundaresan, C. N. R. Rao, J. Phys. Cond. Matter Phys. 21 (2009) 486002.

[6] F. Sugawara, S. Iiida, Y. Syono, S. I. Akimoto, J. Phys. Soc. Japan 25 (1968) 1553.

[7] P. Baettig, C. Ederer, N. A. Spaldin, Phys. Rev. B 72 (2005) 214105.

[8] C. Himcinschi, I. Vrejoiu, T. Weißbach, K.Vijayanandhini, A.Talkenberger, C. Roder, S. Bahmann, D. R. T. Zahn, A. A. Belik, D. Rafaja, J. Kortus, J. Appl. Phys. 110 (2011) 073501; A.Talkenberger, C. Himcinschi, T. Weißbach, K.Vijayanandhini, I. Vrejoiu, C. Roder, S. Bahmann, D. R. T. Zahn, A. A. Belik, D. Rafaja, J. Kortus, Thin Solid Films 520 (2012) 4590.

[9] A. Morelli, F. Johann, N. Schammelt, I. Vrejoiu, Nanotechnology 22 (2011) 265303.

[10] R. Ranjith, R. V. K. Mangalam, Ph. Boullay, A. David, M. B. Lepetit, U. Luders, W. Prellier, A. Da Costa, A. Ferri, R. Desfeux, Gy. Vincze, Zs. Radi, C. Aruta, Appl. Phys. Lett. 96 (2010) 022902, and references therein.

[11] N. Balke, M. Gajek, A. K. Tagantsev, L. W. Martin, Y. H. Chu, R. Ramesh, S. V. Kalinin, Adv. Funct. Mater. 20 (2010) 3466.

[12] F. Zavaliche, R. R. Das, D. M. Kim, C. B. Eom, S. Y. Yang, P. Shafer, R. Ramesh, Appl. Phys. Lett. 87 (2005) 182912.

[13] M. Murakami, S. Fujino, S. H. Lim, C. J. Long, L. G. Salamanca-Riba, M. Wuttig, I. Takeuchi, V. Nagarajan, A. Varatharajan, Appl. Phys. Lett. 88 (2006) 152902.

[14] D. H. Kim, H. N. Lee, M. Varela, H. M. Christen, Appl. Phys. Lett. 89 (2006) 162904.

[15 ] P. Baettig, N. A. Spaldin, Appl. Phys. Lett. 86 (2005) 012505.

[16] N. Ichikawa, M. Arai, Y. Imai, K. Hagiwara, H. Sakama, M. Azuma, Y. Shimakawa, M. Takano, Y. Kotaka, M. Yonetani, H. Fujisawa, M. Shimizu, K. Ishikawa, Y. Cho, Appl. Phys. Express 1 (2008) 101302.

[17] A. A. Belik, S. Iikubo, K. Kodama, N. Igawa, S. Shamoto, E. T. Muromachi, Chem. Mater. 20 (2008) 3765.

[18] A. A. Belik, E. T. Muromachi, J. Phys.: Conf. Series 165 (2009) 012035.

[19] C. Goujon, C. Darie, M. Bacia, H. Klein, L. Ortega, P. Bordet, J. Phys: Conf. Series 121 (2008) 022009. 
[20] S. Niitaka, M. Azuma, M. Takano, E. Nishibori, M. Takata, M. Sakata, Solid State Ionics 172 (2004) 557.

[21] A. David, Ph. Boullay, R. V. K. Mangalam, N. Barrier, W. Prellier, Appl. Phys. Lett. 96 (2010) 221904.

[22] D. G. Schlom, L. -Q. Chen, C. -B. Eom, K. M. Rabe, S. K. Streiffer, J. -M. Triscone, Annu. Rev. Mater. Res. 37 (2007) 589.

[23] D. B. Williams, C. B. Carter, “Transmission electron microscopy”, Plenum Press, New York (1996).

[24] C. J. M. Daumont, S. Farokhipoor, A. Ferri, J. C. Wojdel, J. Iniguez, B. J. Kooi, B. Noheda, Phys. Rev. B 81 (2010) 144115.

[25] C. D. Wagner, W. M. Riggs, L. E. Davis, J. F. Moulder, "Handbook of X-ray Photoelectron Spectroscopy", edited by G. E. Muilenberg, Perkin- Elmer Corp., Eden Prairie (1979).

[26] S. K. Singh, H. Ishiwara, J. Appl. Phys. 100, (2006) 064102; B. H. Park, B. S. Kang, S. D. Bu , T. W. Noh, J. Korean Phys. Soc. 35 (1999) S1306.

[27] K. Rida, A. Benabbas, F. Bouremmad, M. A. Pena, E. Sastre, A. Martinez-Arias, Appl. Catalysis A 327 (2007) 173.

[28] X. Liu, W. Su, Z. Lu, Mater. Chem. Phys. 82 (2003) 327.

[29] P. L Gai, C. N. R. Rao, Pramana 5 (1975) 274.

[30] L. Katz, R. Ward, Inorg. Chem. 3 (1964) 205.

[31] Y. C. Wu, S. F. Wang, S. H. Chen, J. Amer. Ceram. Soc. 92 (2009) 2099

[32] Richard Tilley, "Crystals and crystal structure”, Wiley Publications (2006) 194.

\section{FIGURE CAPTIONS}

Fig. 1. (a) Line graph showing the pseudocubic a-axis lattice constants (in $\AA$ ) for BCO and SRO (above the number line) and NGO and STO substrate (below the number line). ${ }^{[2,22]}$ (b) AFM topography image of a chemically etched and annealed $\mathrm{SrTiO}_{3}(001)$ substrate with the corresponding line scan as inset. (c) AFM surface morphology of a $20 \mathrm{~nm}$ thick $\mathrm{SrRuO}_{3}$ film grown on STO (001). 
Fig. 2. XRD scans for (a) BCOsto and (b) BCONGO films; the (003) reflection is enlarged and shown as an inset.

Fig. 3. (a) - (c) RSM analysis along (203) for BCOsto films with thickness 130 $\mathrm{nm}, 85 \mathrm{~nm}$ and $25 \mathrm{~nm}$, respectively, and (d) RSM for $160 \mathrm{~nm}$ thick BCONGO film.

Fig. 4. AFM morphology of epitaxial $\mathrm{BiCrO}_{3}$ films grown on $\mathrm{SrRuO}_{3}$ buffered (a) $\mathrm{SrTiO}_{3}$ (001) [BCOsto] (RMS roughness: $0.7 \mathrm{~nm}$ ) and (b) $\mathrm{NdGaO}_{3}$ (110) [BCONGO] (RMS roughness: $0.8 \mathrm{~nm}$ ). All the images are $4 \times 4 \mu \mathrm{m}^{2}$. The height scale for both the images is $10 \mathrm{~nm}$.

Fig. 5. XPS data for (a) $\mathrm{Bi} 4 f$, (b) $\mathrm{Cr} 2 p$ and (c) $\mathrm{O}$ 1s core level spectra for a 100 $\mathrm{nm}$ thick BCOsto film. Inset in (a) denotes the enlarged portion of one of the Bimetallic shoulder peaks due to $\mathrm{Ar}^{+}$sputtering (indicated by arrows). Their corresponding Gaussian-Lorentzian fittings (red solid lines) of core level XPS spectra recorded at the BCO film's depth of $22 \mathrm{~nm}$ are depicted in (d), (e) and (f) respectively.

Fig. 6: In-depth XPS analysis that shows the atomic \% with respect to depth of the $\mathrm{BCO}(100 \mathrm{~nm}) / \mathrm{SRO} / \mathrm{STO}$ heterostructure.

Fig. 7. (a) BF image of a $130 \mathrm{~nm}$ thick BCOsto and its corresponding SAED pattern (inset), (b) HRTEM image from the area enclosed by the white box in (a), (c) and (d) FFT patterns from areas marked as 1 and 2 respectively, in (b). The satellite reflections are marked by arrows.

Fig. 8. Plan-view TEM micrograph for the $130 \mathrm{~nm}$ thick BCOsto film. (a) BF image and (b) SAED pattern.

Fig. 9. (a) Overview HAADF-STEM image indicating two dislocation lines enclosed in the white boxes, (b) zoomed-in HAADF-STEM image taken from the dislocation line marked as 1 in (a), and (c) HAADF-STEM image from the top 
dislocation enclosed in the box in (b), showing an edge-type dislocation and its Burgers circuit.

Fig. 10. (a) HAADF-STEM image of BCOSTo film with its corresponding FFT pattern as an inset, indicating the main reflections within the red circle and the extra reflections within three blue circles. b) Filtered image created using the main reflections. c) Filtered image created using the extra reflections. d) False color image combining (b) and (c), with red and blue, respectively.

Fig. 11. Cross section BF TEM images for two BCOsto films with different thicknesses: (a) $85 \mathrm{~nm}$ and (c) $25 \mathrm{~nm}$. The respective SAED patterns for (b) 85 $\mathrm{nm}$ and (d) $25 \mathrm{~nm}$.

Fig. 12. $B_{\text {CO }}$ NG film (S)TEM data: (a) sample overview, (b) high-resolution and (c) SAED pattern. 